\title{
Review on Domestic Lin Shu's Translation Studies in the First Decade of New Century
}

\author{
Ning Li \\ School of Foreign Languages, Guangdong Pharmaceutical University, Guangzhou, PR China
}

Email address:

Glorialeesmile@163.com

\section{To cite this article:}

Ning Li. Review on Domestic Lin Shu's Translation Studies in the First Decade of New Century. International Journal of Applied Linguistics and Translation. Vol. 6, No. 3, 2020, pp. 85-88. doi: 10.11648/j.ijalt.20200603.15

Received: July 31, 2020; Accepted: August 17, 2020; Published: September 3, 2020

\begin{abstract}
As one of the most representative translators on foreign literature in late Qing China, Lin Shu and his foreign novel translations have attracted much attention in the academic field even in nowadays. With the special social background in the late Qing, Lin Shu gradually shifted his focus from western political and historical works to novels in order to express his opinions better. In this study, it will be generalized the domestic research on Lin's translation activity in the first decade of 21 thcentury, which research can be divided into at least four categories, such as the study of Lin's translation ontology problem, discussion of cultural factors of his translations, influential phenomenon of his novel translations and the comparative study on Lin's foreign literature translations. Therefore, the typical case study which points out the various research paradigms as while as further possible avenues of researchon domestic Lin Shu's translation studies in the future.
\end{abstract}

Keywords: Lin Shu, Lin Shu's Translations, Domestic Research

\section{Introduction}

Lin Shu and his foreign literature translations can be treated as one of the highest wonderful works in the translation history of modern Chinese literature. Actually, he himself did not know any of the foreign language at all. Therefore, he evaluated his novel translation activity as "ears are chased by the hands". Most of Lin's translated works were adopted by the method of "rewriting", such as free translation. All through his lifetime, he completed the task of introducing more than 180 foreign literary works into Chinese society, which is obviously made an irreplaceable influence on the development of modern Chinese literature and the improvement of Chinese culture.

In recent years, especially after entering the new century, forest translation study in the field of translations can be carried out in the ascendant with multiple perspectives. There are more methodologies put into effect into Lin Shu's translation studies, such as the analytical discussions on his foreign novel translations, research on his translation activities in the late Qing China, exploring the unique cultural phenomenon of his translated works, analyzing the historical background of Lin's translations, combining modern translation theories with his translation and comparing with
Lin's translation studies in the much broader academic field for the future vision to explore etc.. On the basis of previous research of Lin Shu, the author would like to sort out and classify the studies on Lin's translation activities in the first decade of new century. Moreover, it will try to summarize the perspective into following aspects, respectively are research on the ontology of Lin Shu's translation, the discussion on the cultural issues behind his translation activity, the analysis on the phenomenon of Lin's translation and the comparative research of the contemporary translation phenomena in late Qing China.

\section{Study on Ontology of Lin Shu's Translation}

The research on the ontology of Lin's translation can be included the research on Lin's translation methods, characteristics of his writing style, translation strategies and his motivations of introducing the various types of foreign literary works. One of the most distinctive features of Lin's novel translation method is "interpretation while writing", which means he take down the interpretation with the help of his collaborators as written translation firstly, and then he 
modify the draft by rewriting later on, which is also made scholars interested in Lin Shu's interpretation collaborators. Among the scholars, Guo Yang has introduced part of interpreters of Lin Shu's translation from the perspective of interpreters' influential factors in his research finding. Moreover, Guo also analyzes the process of co-translation and points out the interpreter should not be held fully responsible for the fallacies in Lin's translations [1]. Zhang Xiuyan pays attention to the translation quality and language ability of different interpreters on Lin Shu's translation in the article of The Forgotten Interpreters in Lin Shu's Novel Translations [2], whom also analyzes the advantages and disadvantages of this combination translation mode. Other scholars, such as Wen Yue'e has selected the major collaborator, for example, Wei Yi in Lin's translation to conduct the research finding. Moreover, in the majority of domestic Lin Shu's novel translations studies, the quantity of this aspect is not dominantly, on the contrary, several scattered discourse patterns are in the basic similarity, which needs to be further explored for in-depth analysis of historical materials.

Another distinguishing feature of Lin Shu's translation is that he writes foreign novels with classical Chinese style, which several scholars choose to study Lin's translation from this perspective. For instance, Zhang Zhuxiang and Liu Jiehui take the case study of Lin Shu's translation of $\mathrm{Ba} \mathrm{Li}$ Cha HuaNv Yi Shi as an example to probe into his writing style in the translation press on the acceptation of how to play an important role in the process of ideological evaluation [3]. In the article of Novel Writing: The Resonance and Transformation between Lin Shu's Ancient Writings and Lin's Translated Novels, Wu Wei has paid attention to the convergence of Lin Shu's translated works and creation in the aspect of the conception of "writing". He believes that Lin's "consciously practiced the ancient writings with ancient 'novel writing' style" [4], which has enabled refined and popular literature and Chinese and Western literature to blend together. At the same time, LinShu's faithless translation strategy has also attracted the attention of domestic researchers. Such as, Huang Hanping has indicated in his article "Abridge" and "Supplement" in Literary Translation -- A Cultural Perspective of the Phenomenon of Original Works from the perspective of polysystem for the discussion of Qian Zhongshu's work on Lin Shu's Translation with the review on discourse of Lin's translation by "abridge" and "supplement" in his novel translation from the referential original phenomenon to multi-cultural relations [5]. Besides, by comparing Lin Shu's translation of Haggard's original novels, Liu Hongtao and Liu Qian has discussed Lin's "unfaithfulness" translation to a kind of "creative treason" [6]. Similar through more case studies of Lin Shu's translation strategies and motivations, which includes Yao Yanbo's article Study on Methods of Lin Shu's Translation of Poetic Interpretation, Huang Rong's Study of Lin Shu's Translation on Dickens's Novels, Huang Yanjie's Study of Lin Shu's Translation of Yin Bian Yan Yu and so on. As for this kind of research, due to the large number and diverse types of Lin Shu's translated novels, scholars usually analyze and discuss them through one translationor summarize them in a general way. Therefore, there are some defects in the research, such as duplicity, partial generalization or superficial appearance.

\section{Study on the Cultural Issues Behind Lin Shu's Translation}

With the appearance of "cultural turn" in translation studies, more and more scholars have turned their attentions to the study on the cultural issues behind Lin Shu's translations. Most of them made a diachronic analysis of Lin himself and his translated novels from the perspectives of ideology, cultural choice and literary contradiction. Some of them consider that Lin Shu misunderstood or even adjusted Western literature and culture through subjective efforts into Chinese literary field, while others consider that Lin's translation strategy can be treated as a kind of historical, helpless and contradictory choice during the period of late Qing China.

Refer to the scholars in this research area, which studies have involved in multiple aspects, such as Su Guining's article Study of Cultural Choice in Lin Shu's Novel Translation, in which he has discussed the basic motivation of Lin's translation activity of foreign novels. As meanwhile, he also indicates the perspective of Lin Shu as a turning point of Chinese intellectuals in terms of translation motive, material selection and translation strategies, who has unconsciously showed the ideology of contradiction and selection of literature and art that it is these contradictions prompted Lin Shu's translations as the different cultural tendency in different periods and positioning [7]. Other scholars for example, Ma Bing goes straight to the contradictions of Lin Shu's translations from him excavated in sequence in different periods of the target in his literary ideas, reforming ideas, aesthetics belonging to which of various aspects of ideological contradiction, even describe Lin Shu as the representative paradox of conservative and innovative [8]. However, some scholars do not agree with the description on cultural contradictions or paradox of Lin Shu's translation. On the contrary, they consider Lin's translations have reflected his subjective efforts to adjust conflicts to gain the target readers' acceptance based on his interpretation of western literature into ancient Chinese culture. For example, Yang Hongjun has pointed out that based on the subjective distortion of source language culture, new ideas of western culture and the traditional culture have taken initiative to reconcile for target readers, which have also given the awareness of national identity to the target readers [9]. At this level, Lin Shu's transition of western literature has played the extraordinary role in mental changes in Chinese society at that time. Moreover, with the studies of many scholars, for instance Zhang Juncai, Lin Wei and Li Jingguang whose findings have praised how Lin Shu successfully mediated the conflict between Chinese and Western cultures, even developed the new Chinese literature. While at the same time, other 
researchers have focused on the negative impact of Lin's translation comparing literary reformers then, which consider that Lin's activity in translation, to some extent had delayed the modernization of Chinese literature for his using of cultural paradigm for the target language. In a word, opinions are various within the scope of this study on Lin Shu's translations.

\section{Study on the Cultural Phenomenon of Lin Shu's Translation}

While introducing various western translation theories, Chinese translation circles are also trying to apply these theories to explain typical translation phenomena in Chinese translation history. Therefore, Lin Shu's novels have been regarded as the unique translation phenomenon by most of the domestic scholars; what's more, with the discussion of Lin Shu's typical translation case study, various modern translation theories such as polysystem, postcolonial, manipulation and rewriting theories have been introduced to interpret Lin's foreign novel translations.

In research paper of Study on Lin Shu's Translation from the Perspective of Postcolonial Theory, Liu Yang and Huang Qin have analyzed Lin's translated novels based on the postcolonial translation theory, which is based the social background in late Qing, characteristics of translation selections and translation strategy analysis, while taking the consideration of Lin's translation phenomenon as the third world to face the post-colonialism context. Therefore, various translators would to adopt a new translation criteria and strategy to against the foreign cultural invasion asa kind of subjective efforts [10]. However, in the application of translation theory, this kind of research is often considered as applying the ready-made theories, which with the defects of insufficient analysis and lack of independent thinking and unique insights. At the same time, there are also scholars try to point out the defects and inapplicability of some modern translation theories by analyzing the phenomenon of Lin Shu's translations. For example, in the Study of Lin Shu's Domestication Translation Strategy from the Perspective of Polysystem Theory, Yang Xiaohong has analyzed the adoption of domestication in the translation strategy of internal causes in Lin's novel translated activity [11], which is also pointed out the theory of polysystem would be focused on the objective factors, such as cultural status and subjectivity of translators' to target readers for the specific translation purpose, therefore, which does not apply to explain the special translation phenomenon as Lin Shu's translations.

\section{Comparative Study Between Lin Shu's Translation and Others}

Refer to this point, it belongs to the category of comparative studies. Domestic researchers have put Lin Shu and his foreign literature translation into a much broader academic field in vision, which has also transcended the limitations of time and space, seeking to find similar phenomena in Chinese and Western translation history and make the comparative analysis.

Take Xie Piaoyun's research paper for an example, it has discussed the comparison between Lin Shu's foreign novel translation and Yan Fu's essay translation, which has made detailed descriptions with the influential elements of Lin and Yan's subjectivity [12], such as family background, life time experiences, personal conceptions and emotional personalities, which have held that similarities and differences in these aspects are closely related to their characteristics in translation. Similarly, Zhu Chaowei has taken the comparative study on Lin Shu and Ezra Pound in their translation activities, which through the sorting both of the Chinese and foreign literature in translation thoughts and analysis that have indicated their translation characteristics, such as elegant in rewriting, precise in knowledge information and appropriate in purpose for target reader's appreciation [13]. However, the combination of creative collocation and unfaithful of the original context is also resulted to the negative criticism on their translations in domestic research field. Later on, the "misunderstanding" [14] of Lin Shu and Ezra Pound's translation has gradually become the specific cultural phenomena in translation studies. In addition, some other scholars have compared Lin Shu and the rest similar foreign translators from various perspectives to analyze, among which such as the translator of ancient Chinese literature Arthur Waley [15], which has taken their personal characteristics and subjectivities in Chinese to Western translation activities.

\section{Conclusion}

As one of the most important and well-known modern Chinese translators, Lin shu's contemporary perspective and viewpoint in cultural transformation and social awareness of China in the late Qing and early Republic is obviously impossible to ignore.

Based on the discussions of Lin Shu's foreign translations from at least four aspects above, the review has sorted out and integrated the domestic research perspectives in the first decade of new century, nevertheless, due to the limited original material and the space limitation, the review only has presented the list of some typical examples to illustrate the related research achievements on types of context, developed direction, perspectives that can be adopted and to some extent, the existing limitations in Lin Shu's translation studies, which has expected to make future study and exploration in this research area.

\section{Acknowledgements}

This Article Is Funded by 2018 Young Innovative Talents Project of Guangdong Entitled "Study on the Narrative of National Imagination in the Late Qing China Based on the Cultural Field" (2018WQNCX062). 


\section{References}

[1] Guo Yang. An Examination of Lin Shu's Interpreters in Novel Translations [J]. Chinese Literature Studies, 2008 (4): 42.

[2] Ma Zuyi. A Brief History of Translation in China [M]. Shanghai: Shanghai Foreign Language Teaching Press, 1998.

[3] Zha Jian, Xie Tianzhen. Translation History of Chinese Foreign Literature in the 20th Century [M]. Wuhan: Hubei Education Press, 2007.

[4] Huang Hanping. "Abridge" and "Supplement" in Literary Translation -- Cultural Perspective of the Phenomenon of Original Works [J]. China Translation, 2003 (4): 77.

[5] Huang Yanjie. Case Study on Interpretation of Yin Bian Yan $\mathrm{Yu}[\mathrm{J}]$. Journal of Tianjin Foreign Languages University, 2008 (4): 68.

[6] Liu Hongtao, Liu Qian. On Creative Treason in Lin Shu's Translated Novels [J]. Journal of Beijing Normal University, 2008 (3): 105.

[7] Liu Yang, Huang Qin. Analysis of Lin Shu's Translation from the Perspective of Postcolonial Translation Theory [J]. Journal of Huazhong University of Science and Technology, 2010 (3): 159.

[8] Ma Bing. Contradiction between Lin Shu and Pioneers of "May 4th"Literary Movement in Similarities and Differences [J]. Dong Yue Treatise, 2003 (1): 129.

[9] Su Guining. Lin Shu's Translated Novels and His Cultural Choice [J]. Literary Review, 2000 (5): 60-67.

[10] WenYue'e. Study on the Co-translation of Wei Yi and Lin Shu [J]. Journal of Hunan Agricultural University, 2008 (3): 78.

[11] Wu Wei. Novel Style: Resonance and Transformation between Lin Shu's Ancient Prose and Lin's Translated Novels [J]. Novel Research in Ming and Qing Dynasties, 2002 (3): 117.

[12] Yang Hongjun. On the Cultural Characteristics of Lin Shu's Novel Translations [J]. Journal of Zhaoqing University, 2006 (1): 61 .

[13] Yao Yanbo. Poetic Interpretation of Translation method of Lin Shu's Hei $\mathrm{Nu} \mathrm{Yu} \mathrm{Tian} \mathrm{Lu} \mathrm{[J].} \mathrm{Journal} \mathrm{of} \mathrm{Zhejiang} \mathrm{Ocean}$ University, 2007 (4): 69.

[14] Zhu Chaowei. Comparative Studies on Translation Thoughts between Lin Shu and EzraPound's Translations [J]. Journal of PLA Foreign Languages Institute, 2002 (3): 101.

[15] Wang Guimei. The Silence of the Old School and Lin Shu's Situation: The May 4th Cultural Controversy between the Old and the New in 1919 [J]. China University Academic Abstracts, 2019 (3): 216. 